\title{
Paranoid Personality Masking an Atypical Case of Frontotemporal Dementia
}

\author{
Nneka Iroka $^{a}$, Waqas Jehangir ${ }^{\mathrm{a}, \mathrm{d}}$, Jay Littlefield II $^{\mathrm{b}}$, Vishwanath Pattan ${ }^{\mathrm{a}}$, Abdalla Yousif ${ }^{\mathrm{c}}$, Arunesh K Mishra $^{\mathrm{a}}$
}

\begin{abstract}
Frontotemporal dementia (FTD) is a debilitating disease that is well described in the "Diagnostic and statistical manual of mental disorders, fifth edition (DSM-5)", and typically presents with memory impairment, progressive decline in cortical functioning, and behavioral changes. Age of onset is generally in the late fifties, and usually the first presentation involves a change in behavior and emotional blunting. Treatment of FTD involves management of any neurobehavioral symptoms while trials of atypical antipsychotics are ongoing but suggest some efficacy. We present a case of a patient who first presented with severe paranoid personality traits and frank persecutory delusions. This atypical presentation of our patient first led to her incorrect diagnosis of a psychotic disorder and paranoid personality disorder. As a result of this diagnosis, she was treated unsuccessfully. A subsequent magnetic resonance imaging (MRI) then showed atrophy of frontal and temporal lobes bilaterally (left more prominent than right) which confirmed the diagnosis of FTD. The importance of this case involves the atypical presentation of paranoia and delusions, and our patient's incorrect diagnosis based on her clinical presentation led to a trial of unsuccessful treatment. Only after performing an MRI, which showed atrophy, was the patient appropriately treated and deemed medically stable. This case report illustrates the importance of considering a rare presentation of frontotemporal lobe dementia with patients who are in the typical age range and present with paranoia and delusions.
\end{abstract}

Keywords: Frontotemporal dementia; Paranoia; Delusions

\section{Introduction}

Dementia is a debilitating disease of the elderly with impair-

Manuscript accepted for publication January 28, 2015

${ }^{a}$ Internal Medicine Residents, Raritan Bay Medical Center, 530 New Brunswick Avenue, Perth Amboy, NJ 08861, USA

${ }^{b}$ Ross University School of Medicine, Dominica

cRaritan Bay Medical Center, Perth Amboy, NJ, USA

${ }^{\mathrm{d} C o r r e s p o n d i n g ~ A u t h o r: ~ W a q a s ~ J e h a n g i r, ~} 530$ New Brunswick Ave, Perth Am-

boy, NJ 08861, USA. Email: wjehangir@hotmail.com

doi: http://dx.doi.org/10.14740/jocmr2099w ment in memory, cognition, and progressively worsening cortical functioning [1]. Frontotemporal dementia (FTD) is a relatively rare form of dementia that is well described in the "Diagnostic and statistical manual of mental disorders, fifth edition (DSM-5)", and typically presents with memory impairment, progressive decline in cortical functioning, and behavioral changes. It is a relatively uncommon disease and most often misdiagnosed as a psychiatric disorder. The age of onset is generally in the late fifties, with a distribution among males and females accounted for $66 \%$ and $34 \%$ of the cases, respectively [2]. Often, one of the first presentations of FTD involves a change in behavior and emotional blunting [3]. Diagnosis of less common variants of dementia with atypical symptoms can be a diagnostic challenge and may mislead clinicians to do extensive investigational workup with prolonged hospitalizations resulting in a delay of patient treatment, and a greater economic burden. In this paper, we will present an uncommon and atypical presentation of dementia in a patient who otherwise would not be included in the differential diagnosis because of her normal mini-mental status examination. We report a case of FTD in a 57-year-old female who was examined and was diagnosed with delusional disorder and paranoia. This case illustrates the importance of considering FTD in relatively young patients presenting with signs and symptoms of rapid behavioral changes such as delusions and paranoia.

\section{Case Report}

A 57-year-old female was brought to the emergency room by her daughter because the patient was exhibiting signs and symptoms of paranoia and frank persecutory delusions for the past year. She believed that her next door neighbor was watching her and listening to all of her conversations. She also believed that her neighbor had wired the house and that she was being monitored. She had a past history of paranoid personality but has been functional and socially active and had worked for an insurance company as a senior adjuster for about 30 years until 2 years earlier when she was laid off. She is divorced and has two grown children. She had no previous psychiatric admission. Her past medical history was significant for hypertension which was well controlled. Physical examination finding showed blood pressure $135 / 82 \mathrm{~mm} \mathrm{Hg}$, respiration rate $20 / \mathrm{min}$, pulse $85 / \mathrm{min}$, oxygen saturation on room air $100 \%$, and tem- 


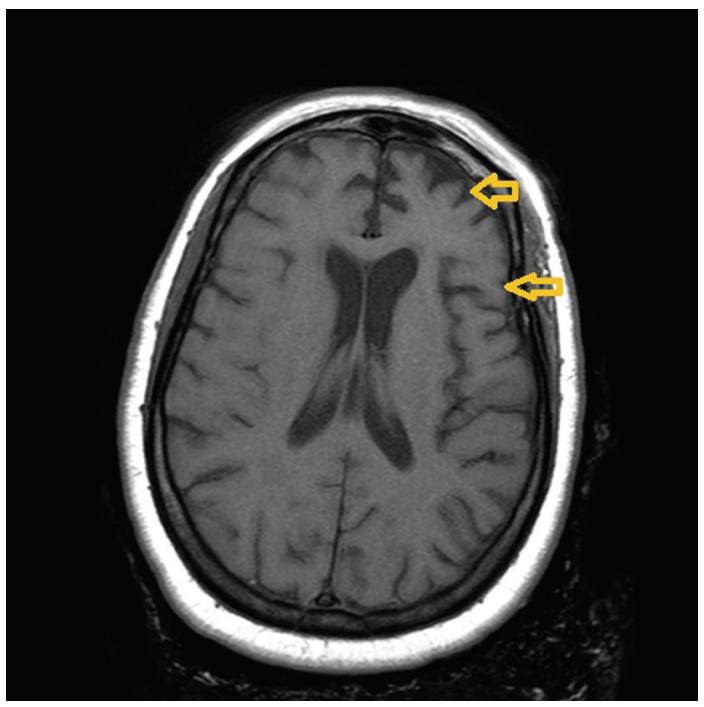

Figure 1. Atrophy of frontal and temporal lobes bilaterally left more prominent than right.

perature $99.4^{\circ} \mathrm{F}$. She was neatly dressed but was maloderous. On mental status examination, she scored 14 on the Montreal cognitive assessment test. Memory and mini-mental status exam was unremarkable. Other than speech fluency that was reduced, the rest of her physical examination was unremarkable. A complete blood count and complete metabolic profile were within normal limits. Vitamin B12 and thyroid stimulating hormone levels were within normal limits. A computerized tomography (CT) scan of the brain was unremarkable. A diagnosis of paranoid and delusional disorder non-otherwise specified was made, and she was started on olanzapine $2.5 \mathrm{mg}$ daily and was considered for discharge home. The daughter at this time further expressed concerns for safety as she said the patient sometimes forget to pay her utility bills and that her house might be without electricity. At this point, with the new information of the patient's inability to care for herself, a diagnosis of FTD was considered, taking into account her age and the time frame of her symptoms. The patient was admitted to the hospital, and a subsequent magnetic resonance imaging (MRI) of the brain was obtained which showed atrophy of frontal and temporal lobes bilaterally, left more prominent than right, which confirmed the diagnosis of FTD (Fig. 1, 2). The patient was appropriately treated thereafter with olanzapine 10 $\mathrm{mg}$ daily and $5 \mathrm{mg}$ as needed for agitation, and donepezil 5 $\mathrm{mg}$ at night as well. She improved symptomatically and was later discharged home to her family in stable condition with appropriate follow-up.

\section{Discussion}

FTD is the fourth most common cause of dementia after Alzheimer's dementia, dementia with lewy bodies and vascular dementia. It is a relatively uncommon form of dementia; however, it generally is associated with an earlier age of onset, as well as a progressive change or decline in personality, social

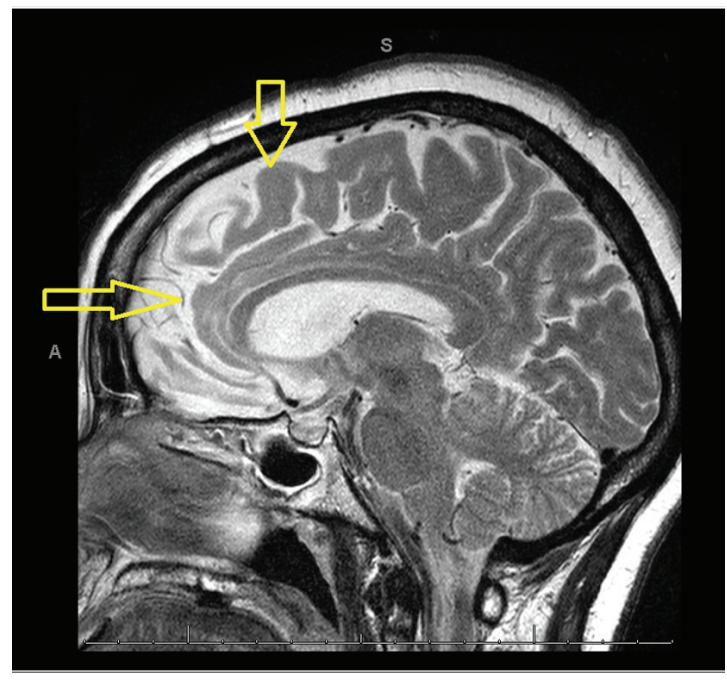

Figure 2. Atrophy of frontal and temporal lobes of right side.

behavior, and language [4]. FTD may account for $20-50 \%$ of dementia cases in people younger than age 65 , which one study found was very similar in incidence to Alzheimer's dementia in the same subgroup of patients [5]. It was first described in 1892 by Arnold pick in a patient with distinct symptoms affecting language. There are different spectra of FTD; however, the behavioral variant FTD, which is the most common, takes its greatest toll on personality and behavior [6]. It may present with subtle changes that may be mistaken for depression. As it progresses people often developed disinhibition, a striking loss of restraint in personal relations and social life. Supportive diagnostic features include a decline in personal hygiene, mental rigidity [7]. This is where the differences were in the case described. Our patient presented differently from the common presentation of FTD. The paranoia and the persecutory delusions did not fit this classic presentation of change in behavior and social disinhibition, and actually steered the diagnosis away from FTD towards a paranoid personality disorder. The changes in personality, decrease amount of speech, and the paranoia and persecutory delusions all support the diagnosis of behavioral variant FTD.

Diagnosis of FTD is made primarily by clinical assessment. Neuroimaging studies are required to exclude alternative pathologies, reversible causes of dementia, and may provide supporting findings. Progressive aphasia is also a supportive finding of FTD in patients who have more advanced temporal lobe involvement [8]. These imaging studies can be used to rule out other causes of dementia, and can help to strengthen the clinical diagnostic criteria of FTD. A brain MRI is performed to exclude any structural disease, and confirm FTD with focal or general atrophy of the frontal and/or temporal lobes [9-11]. It was only after performing the MRI, which showed the bilateral atrophy of the temporal lobes (Fig. 1), and the frontal lobe (Fig. 2), that the definitive diagnosis of FTD was made.

Differentiating behavioral variant FTD from Alzheimer's dementia is best done through reporting of behavior from family members or care-givers. The patients themselves are rarely a reliable source of information. The general consensus from 
the literature on the diagnostic behavior of FTD includes the presence of personality change, decline in personal hygiene, change in personal and social relationships, delusions, progressive aphasia, and mental competency $[9,12,13]$. Our patient was fortunate to have a daughter who was so in tune to her situation. Without this valuable source of information, it is an even higher likelihood that an incorrect diagnosis and treatment will occur.

Treatment of FTD is symptomatic and is generally aimed at alleviating neurobehavioral symptoms. Pharmacological interventions are currently being used in the treatment of FTD; however, there is limited evidence of the efficacy of these medications. Atypical antipsychotic medications, such as olanzapine, such as was used in to successfully treat our patient, can help to decrease delusions and other neurobehavioral symptoms in FTD [14]. Serotonin selective reuptake inhibitors have also been reported to decrease impulsivity and anxiety [15]. In addition to pharmacological agents, nonpharmacologic intervention is also important to focus on health maintenance and safety.

Diagnosis of FTD is mainly clinical but MRI of the brain often plays a key role. However misdiagnosis of FTD can be seen when patients present with atypical features. This case illustrates that FTD can present with atypical signs and symptoms such as paranoia and delusions and having a high index of suspicion in the relevant setting thus becomes imperative for appropriate diagnosis and avoidance of improper management.

\section{Conflict of Interest}

The authors declare that there is no conflict of interests regarding the publication of this article.

\section{References}

1. Reisberg B, Ferris SH, de Leon MJ, Crook T. The Global Deterioration Scale for assessment of primary degenerative dementia. Am J Psychiatry. 1982;139(9):1136-1139.

2. Westbury C, Bub D. Primary progressive aphasia: a review of 112 cases. Brain Lang. 1997;60(3):381-406.

3. Rascovsky K, Hodges JR, Knopman D, Mendez MF, Kramer JH, Neuhaus J, van Swieten JC, et al. Sensitivity of revised diagnostic criteria for the behavioural variant of frontotemporal dementia. Brain. 2011;134(Pt 9):24562477.

4. Clinical and neuropathological criteria for frontotemporal dementia. The Lund and Manchester Groups. J Neurol Neurosurg Psychiatry. 1994;57(4):416-418.

5. Mercy L, Hodges JR, Dawson K, Barker RA, Brayne C. Incidence of early-onset dementias in Cambridgeshire, United Kingdom. Neurology. 2008;71(19):1496-1499.

6. Liu W, Miller BL, Kramer JH, Rankin K, Wyss-Coray C, Gearhart R, Phengrasamy L, et al. Behavioral disorders in the frontal and temporal variants of frontotemporal dementia. Neurology. 2004;62(5):742-748.

7. Eslinger PJ, Dennis K, Moore P, Antani S, Hauck R, Grossman M. Metacognitive deficits in frontotemporal dementia. J Neurol Neurosurg Psychiatry. 2005;76(12):16301635 .

8. Grossman M. The non-fluent/agrammatic variant of primary progressive aphasia. Lancet Neurol. 2012;11(6):545555.

9. Kertesz A, Davidson W, Fox H. Frontal behavioral inventory: diagnostic criteria for frontal lobe dementia. Can J Neurol Sci. 1997;24(1):29-36.

10. Pasquier F, Fukui T, Sarazin M, Pijnenburg Y, Diehl J, Grundman M, Miller BL. Laboratory investigations and treatment in frontotemporal dementia. Ann Neurol. 2003;54(Suppl 5):S32-35.

11. Rohrer JD, Warren JD, Modat M, Ridgway GR, Douiri A, Rossor MN, Ourselin S, et al. Patterns of cortical thinning in the language variants of frontotemporal lobar degeneration. Neurology. 2009;72(18):1562-1569.

12. Bathgate D, Snowden JS, Varma A, Blackshaw A, Neary D. Behaviour in frontotemporal dementia, Alzheimer's disease and vascular dementia. Acta Neurol Scand. 2001;103(6):367-378.

13. Kertesz A, Blair M, McMonagle P, Munoz DG. The diagnosis and course of frontotemporal dementia. Alzheimer Dis Assoc Disord. 2007;21(2):155-163.

14. Moretti R, Torre P, Antonello RM, Cazzato G, Griggio $\mathrm{S}$, Bava A. Olanzapine as a treatment of neuropsychiatric disorders of Alzheimer's disease and other dementias: a 24-month follow-up of 68 patients. Am J Alzheimers Dis Other Demen. 2003;18(4):205-214.

15. Swartz JR, Miller BL, Lesser IM, Darby AL. Frontotemporal dementia: treatment response to serotonin selective reuptake inhibitors. J Clin Psychiatry. 1997;58(5):212216. 